\title{
The Polymorphism and Transformation of (3aRS, 4RS, 7RS, 7aSR)-2-(Tricyclo[3.3.1.13,7]decan-1-yl)-4,5,6,7- tetrahydro-4,7-eposyisoindoline-1,3-dione (SU2162)—A Novel Anticancer Compound
}

\author{
Yuqing Huang, Zaiyou Tan*, Lin Luo, Hui Yang, Chunmei Tong, Wenfei Chen, Tingyu Huang, \\ Ruiling Liu
}

School of Pharmacy, Guangdong Pharmaceutical University, Guangzhou, China.

Email: tanzaiyou@medmail.com.cn

Received October $4^{\text {th }}, 2013$; revised November $4^{\text {th }}, 2013$; accepted November $11^{\text {th }}, 2013$

Copyright (C) 2014 Yuqing Huang et al. This is an open access article distributed under the Creative Commons Attribution License, which permits unrestricted use, distribution, and reproduction in any medium, provided the original work is properly cited. In accordance of the Creative Commons Attribution License all Copyrights (C) 2014 are reserved for SCIRP and the owner of the intellectual property Yuqing Huang et al. All Copyright (C) 2014 are guarded by law and by SCIRP as a guardian.

\section{ABSTRACT}

Objective: To determine the transformation between two known crystal forms of the title $\mathrm{Compound}_{(\mathrm{C}} \mathrm{C}_{18} \mathrm{H}_{23} \mathrm{NO}_{3}$, $\mathrm{Mr}=301.37)$. Methods: To recrystallize or heat the crystals and determine the crystal form by testing the melting points. Results: Both the two known crystal forms of the title compound can be changed by dissolving into different organic solvents such as acetone and ethyl acetate. Crystal form I was not influenced by heating while crystal form II can be transformed to crystal form I through melting method. Conclusion: Organic solvents have significant influences on the two crystal forms of title compound. Crystal form I shows a better thermal stability than crystal form II.

\section{KEYWORDS}

\section{SU2162; Polymorphism; Crystal Form; Melting Method; Solvent Method}

\section{Introduction}

In recent study, pharmaceutical cocrystals have shown that the active pharmaceutical ingredient (API) in different forms could present a different solid form and the different form could have different physical and chemical properties, even the therapeutic properties. Identifying the optimum solid form of a pharmaceutical candidate and increasing the efficiency of API screening required the fully understanding of the different crystals from the API. And moreover, the Food and Drug Administration's (FDA) process of efficient manufacturing technologies and process of analytical technology (PAT) could timely measure the quality and performance attributes of raw and in-process materials and products [1]. The implication of PAT had already shown its values in the crystallization processes [2]. Compared with other classes of

"Corresponding author. solid forms, cocrystals possess particularly scientific and regulatory advantages, and alongside these advantages there are intellectual property issues which confer cocrystals with unique opportunities and challenges. Cocrystallization provides alternative solid-state modifications of APIs [3].

According to the world health organization report, data show that there are 9 million new cancer cases every year, and 5 million people die of cancer. Due to the extension of human life expectancy, lifestyle changes and the causes of smoking, if there are no further measures, by 2020 it will have 20 million new cancer cases every year, and the death toll will break through 10 million. The American Cancer Society [4] combining the cancer incidence, mortality, and survival rate from Cancer Institute (NCI) and the centers for disease control and prevention (CDC) forecasts that the United States will have 1,660,290 new cancer cases, and 580,350 patients will die due to 
cancer in 2013.

Norcantharidin, the first synthesis of novel anticancer drugs, is replacing the 3, 4 methyl on cantharidin with hydrogen atom to form cantharidin derivatives. It is a white crystalline powder, odourless and stimulating. And it shows the acidic aqueous solution, slightly soluble in water and ethanol, soluble in hot water and acetone. Norcantharidin, furan and maleic anhydride as raw materials, on the basis of the Diels-Alder addition reaction, get hydrogen to armor cantharidin and catalytic hydrogenation. Synthetic route is shown in Figure 1.

Norcantharidin keeps not only the stronger antitumor activity and also the unique role of elevated white blood cell. Cantharidin can significantly reduce a strong irritant of the urinary system, and is suitable for the treatment of primary liver cancer. Norcantharidin, however, there is still certain viscera toxicity causing the limitation of administration dose in clinical practice, which affects the anticancer effect. People deeply optimize its structure, and strive to further reduce its toxic side effects, which is the same situation of norcantharidin imidization modification. In addition, the study found that the chronic hepatitis b virus (HBV) infection is closely related to the occurrence of cancer of the liver, and amantadine has antiviral effect. Thus, Professor Tan Zai-you used de-amantadine norcantharidin imidated modification and got Norcantharidin amantadine derivatives (SU2162). Its chemical name is (3aRS, 4S, 7R, 7aS)-4,7-epoxy-hexahydro-2-(tricycle [3.3.1.13,7]decane)-1H-isoindole-1,3(2H)-dione, and the international number is SU2162. Structural formula is shown in Figure 2. Professor Tan Zai-you was granted the patent, "an anti-tumor activity of cantharidin derivatives and their preparation methods" (Patent No.: ZL2007 1 0029736.1) [5] on May 7, 2008 by China State Intellectual Property Office.

Professor Tan Zai-you etc. [6,7] studied the crystalline compound and found that the compound has two kinds of crystal structure. Lattice parameters are as follows: crystal form I, is monoclinic, mp $159^{\circ} \mathrm{C}-161^{\circ} \mathrm{C}$, the unit cell contains one molecule, cell volume 1507.35 (6) $\wedge 3$, a = 6.87288 (16) $\AA, \mathrm{B}=10.2647$ (2) $\AA, \mathrm{c}=21.4346$ (5) $\AA, \alpha$ $=90^{\circ}, \beta=94.579(2), \gamma=90^{\circ}$; crystal form II, is triclinic, mp $165^{\circ} \mathrm{C}-166^{\circ} \mathrm{C}$, the unit cell contains six molecules, cell volume 1507.35 (6) $\wedge 3, \mathrm{a}=12.2216$ (4) $\AA, \mathrm{B}=$ 12.3465 (4) $\AA, \mathrm{c}=16.1646$ (6) $\AA, \alpha=77.057$ (4); $\beta=$ 89.906 (3); $\gamma=69.190$ (4).

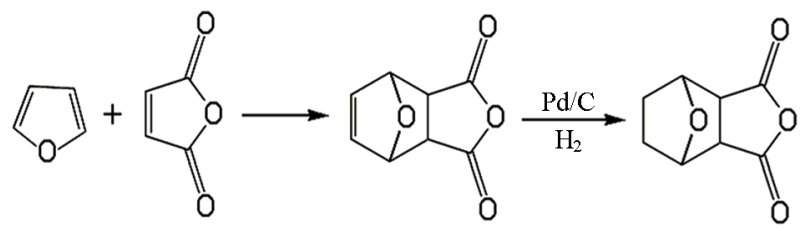

Figure 1. The synthesis route of norcantharidin.
The title compound, an adamantane derivative of norcantharidin, is a novel compound with high anticancer activity and low toxic side effects [5]. Two crystal forms of this compound are belong to triclinic and monoclinic crystal systems respectively. They have been found and reported by our research group before $[6,8]$. In this paper, the transformation of the two known crystal forms confirmed recently was reported.

\section{Experimental}

\subsection{Materials and Physical Measurements}

Norcanthardin (CP), adamantine hydrochloride (CP), methlene chloride (AP), acetone (AP), tetrahydrofuran (AP), ethyl acetate (AP), absolute ethyl alcohol (AP).

Micro Melting Point Apparatus (X-6), DTA (XR-2R).

\subsection{Preparation of Polymorphs}

\subsubsection{Preparation of Crystal Form I}

Norcanthardin (1.0 g) and adamantine (0.9 g) were dissolved in DMF (10 mL) and mixture was heated to reflux with stirring for $18 \mathrm{~h}$. The solvent was then evaporated off and the crude product remaining was dissolved in warm acetone $(10 \mathrm{~mL})$ and cooled rapidly. The clear solution obtained was left undisturbed at $255 \mathrm{~K}$ for several days and gave finally colourless crystals (I).

\subsubsection{Preparation of Crystal Form II}

A mixture of norcanthardin (1.0 g), adamantine (0.9 g) and DMF $(10 \mathrm{~mL})$ was heated to reflux with stirring for $18 \mathrm{~h}$. The solvent was removed by evaporation and the crude product remaining was dissolved in ethyl acetate (10 mL). The clear solution obtained was left undisturbed for two days. Then the ethyl acetate was evaporated and the residue was washed several times with $20 \mathrm{~mL}$ boiled ethanol water (ethanol:water $=1: 4$ ). After recrystallization from ethyl acetate at room temperature, well-defined pure-white crystals (II) were obtained.

\subsection{Transformation of the Two Crystal Forms}

\subsubsection{Recrystallization}

Crystals (I, 0.2 g) were dissolved in warm ethyl acetate

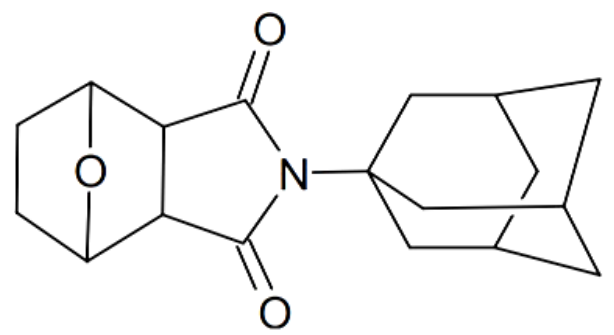

Figure 2. Chemical structure of SU2162. 
(2 $\mathrm{mL}$ ) and cooled rapidly. The clear solution obtained was left undisturbed at $255 \mathrm{~K}$ for several days and gave finally colourless crystals (I'). Crystals (II, 0.2 g) were dissolved in warm acetone $(2 \mathrm{~mL})$ and cooled rapidly. The clear solution obtained was left undisturbed at $255 \mathrm{~K}$ for several days and gave finally colourless crystals (II').

\subsubsection{Melting Method}

Crystals (I, $10 \mathrm{mg}$ ) and crystals (II, $10 \mathrm{mg}$ ) were placed into the alumina crucibles and the thermal analysis curves were tested through DTA respectively (heating rate: $10^{\circ} \mathrm{C} / \mathrm{min}$, temperature range: room temperature $260^{\circ} \mathrm{C}$ ). After the molten crystals cooling, the thermal analysis curves were tested in the same operating conditions again respectively. Then, all the thermal analysis curves were analysed and compared.

\section{Result and Discussion}

\subsection{Recrystallization}

The melting points of crystals I, II, I' and II' were tested by the Micro Melting Point Apparatus, and the results were as follows (Table 1 ).

The figures above indicate that the organic solvents have crucial influence on the crystal forms of the title compound. The two known crystal forms can be transformed mutually by selectively dissolving the crystals into acetone or ethyl acetate.

According to the solvent system [8], we used lots of different solvent to recrystallize the compound and finally found this appropriate solvent to get the different crystal forms.

\subsection{Melting Method}

The thermal analysis curves of crystal (I) are as follows (Figure 3).

The results of the thermal analysis illustrate that the melting point of crystal (I) was not changed remarkably by heating, meaning that the crystal form I has good thermal stability.

The thermal analysis curves of crystal (II) are as follows (Figure 4).

Comparing with the result of initial test, the melting point of crystal (II) significantly declined to that of crys-

Table 1. Melting point of crystals.

\begin{tabular}{cc}
\hline Sample & Melting Point $/{ }^{\circ} \mathrm{C}$ \\
\hline I & $165.2-166.4$ \\
II & $160.1-161.3$ \\
I' & $159.3-160.9$ \\
II' & $165.4-166.1$ \\
\hline
\end{tabular}
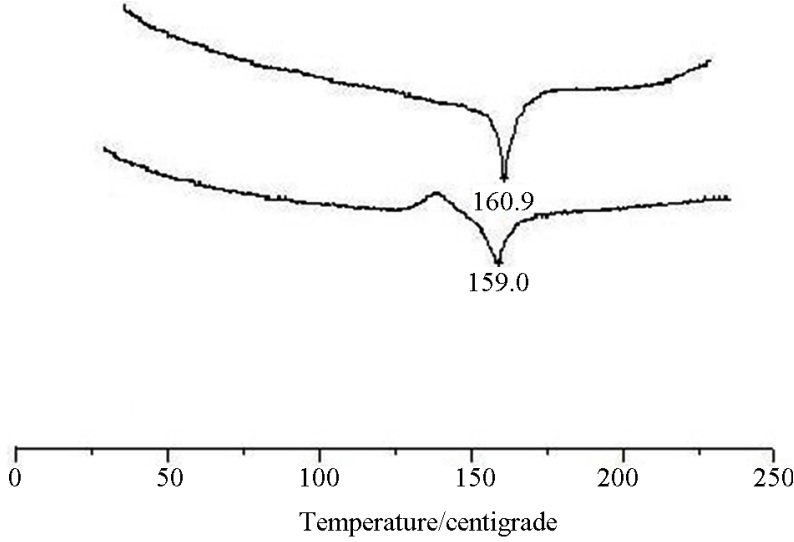

Figure 3. The thermal analysis curves of crystal (I).
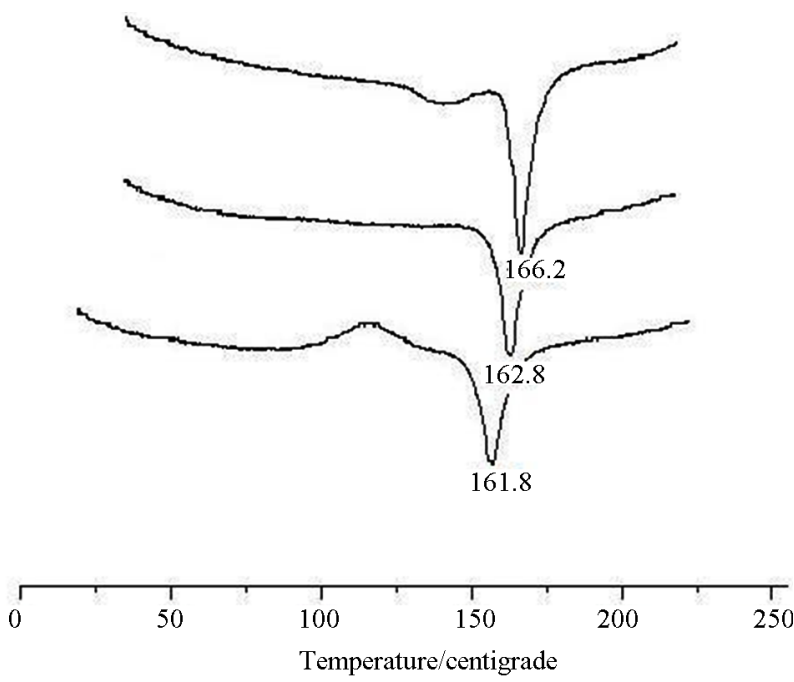

Figure 4. The thermal analysis curves of crystal (II).

tal (I) in the other two tests. It indicated that crystal form II can be transformed to crystal form I by heating.

Through recrystallization and melting method, it has been confirmed that the crystal form of the title compound can be affected by both organic solvents and heating. This method will be applied in our lab to provide a basis for new drug research. At the same time, it can provide guidance for screening materials. Therefore, it is essential for subsequent study.

\section{Conclusions}

An appropriate crystal form for a new chemical provides the opportunity to modify the characteristics of the potential drug substance and to permit the development of dosage forms with good bioavailability, stability, manufacturability, and reduces the side affect. Different crystal forms have different physical chemistry profiles. For instance, the dissolution of a polymorphs drug in vivo determines the extent to which a drug is available for 
absorption dependent upon the crystal form of the drug.

In the current work, a detailed transformation condition between the two crystal forms of the title compound is clear. For further studying the difference of the absorption, distribution, metabolism and its efficacy and biological activity of two crystal forms, this work provided an available method to conveniently convert the crystal form.

\section{REFERENCES}

[1] FDA, "FDA PAT Page." http://www.fda.gov/cder/OPS/PAT.htm

[2] L. X. Yu, R. A. Lionberger, A. S. Raw, et al., “Applications of Process Analytical Technology to Crystallization Processes," Advanced Drug Delivery Reviews, Vol. 56, No. 3, 2004, pp. 349-369. http://dx.doi.org/10.1016/j.addr.2003.10.012

[3] A. V. Trask, "An Overview of Pharmaceutical Cocrystals as Intellectual Property,” Molecular Pharmacology, Vol. 4, No. 3, 2007, pp. 301-309. http://dx.doi.org/10.1021/mp070001z
[4] R. Siegel, D. Naishadham, A. Jemal, et al., "Cancer Statistics, 2013,” CA: A Cancer Journal for Clinicians, Vol. 63, No. 6, 2013, pp. 11-30. http://dx.doi.org/10.3322/caac.21166

[5] Z.-Y. Tan, K. Liang, H. Zhang, et al., "Preparation of a Derivative of Cantharidin with Anticancer Activity,” China Patent No. 200710029736, 2008/

[6] Z.-Y. Tan, L. Luo, E.-J. Zhu, et al., "Preparation and XRay Diffraction Analysis of a New Crystal Form of (3aRS, 4SR, 7RS, 7aSR)-2-(Tricyclo [3.3.1.13,7]decan1-yl)-4,5,6,7-tetrahydro-4,7-eposyisoindoline-1,3-dioneA Novel Anticancer Compound," Journal of Guangdong Pharmaceutical College, Vol. 26, No. 6, 2010, pp. 572575.

[7] Z.-Y. Tan, E.-J. Zhu, L. Luo, et al., “A Method of Inhibiting Platelet Aggregation, Anti-Thrombosis Ferulic Acid Crystal and Preparation Method,” TMP: China Patent No. ZL201010562477.0, 2013.

[8] Z.-Y. Tan, L. Luo, E.-J. Zhu, et al., “(3aRS, 4RS, 7RS, 7aSR)-2-(Tricyclo[3.3.1.13,7]decan-1-yl)-4,5,6,7-tetrahy dro-4,7-eposyisoindoline-1,3-dione,” Acta Crystallographica, Vol. E66, 2010, Article ID: 01374. 